\title{
Diffuse Interface Model for Microstructure Evolution
}

\section{Britta Nestler}

Karlsruhe University of Applied Sciences, Moltkestrasse 30, 76133 Karlsruhe, Germany britta.nestler@fh-karlsruhe.de

Abstract. A phase-field model for a general class of multi-phase metallic alloys is proposed which describes both, multi-phase solidification phenomena as well as polycrystalline grain structures. The model serves as a computational method to simulate the motion and kinetics of multiple phase boundaries and enables the visualization of the diffusion processes and of the phase transitions in multi-phase systems. Numerical simulations are presented which illustrate the capability of the phase-field model to recover a variety of complex experimental growth structures. In particular, the phase-field model can be used to simulate microstructure evolutions in eutectic, peritectic and monotectic alloys. In addition, polycrystalline grain structures with effects such as wetting, grain growth, symmetry properties of adjacent triple junctions in thin film samples and stability criteria at multiple junctions are described by phase-field simulations.

\section{Introduction}

The microstructure formation in metallic alloys involves several different phases and phase transformations. The process is influenced by a great variety of external conditions and physical quantities, e.g. mass and heat diffusion, convection, anisotropy, elasticity etc. and takes place on different time and length scales, see Fig. 1.

Therefore the solidification process of materials yields complex interfacial growth structures and changes in growth topology. The characteristics of the grown microstructure such as the fineness, type of morphology and the spacings themselves determine the physical and mechanical properties of the casting and are hence of great importance with respect to a continuous improvement and optimization of experimental and industrial procedure aiming to produce materials with specific properties. Since solidification in metallic alloys can not in-situ be observed, either experiments on transparent organic substances or modelling methods and numerical simulations are used in order to systematically investigate the influence of the process conditions and of the material properties on the microstructure characteristics.

Traditionally, phase transitions have been expressed mathematically by free boundary problems, where the interface is represented by a sharp surface

B. Kramer (Ed.): Adv. in Solid State Phys. 42, pp. 207-218, 2002.

(C) Springer-Verlag Berlin Heidelberg 2002 

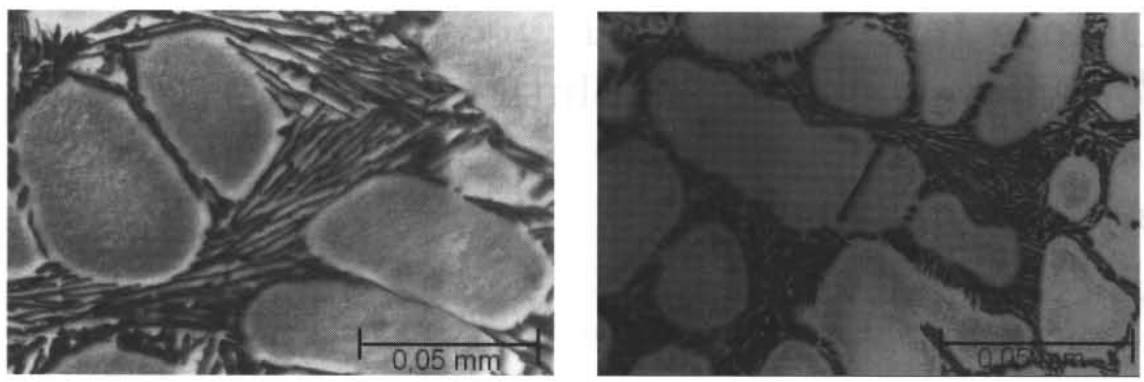

Fig. 1. Examples of the microstructure in a binary Al-Si alloy showing primary $\mathrm{Al}$-dendrites and interdendrititic eutectic regions. Due to the facetted nature of the silicon, the eutectic occurs in an irregular needle-like growth mode

of zero thickness. In these sharp interface formulations the position of the interface has to be calculated explicitly, which leads to great difficulties in the computational treatment of the free boundary problem. In order to handle the moving free boundary numerically, diffuse interface models, e.g. phasefield models have been developed, where the interface is expressed implicitly by a time and space dependent function indicating the phase state and being defined on the whole region. In the last decade, phase-field models have attracted considerable importance as a means of describing and numerically simulating a range of phase transitions and the temporal evolution of complex growth structures that occur during solidification in alloys. Besides the investigation of phenomena on individual length scales, an important challenge for future work on phase-field modelling will be to extend the method so that it can be applied to multiscale problems.

In the standard phase-field description for solid/liquid phase systems, a variable $\phi(\boldsymbol{x}, t)$, called the phase field is introduced whose value characterises the phase of the system in time and space. The interfaces are represented by thin regions in which $\phi$ varies smoothly between the values of $\phi$ associated with the adjoining bulk phases. The mathematical model is based on a free energy functional

$$
\mathcal{F}(\phi, c)=\int_{V}\left(\frac{1}{2} \eta^{2}|\nabla \phi|^{2}+f(\phi, c)\right) d V,
$$

where $f(\phi, c ; T)$ is typically of the form of a double well potential. A set of governing partial differential equations for the appropriate thermodynamic quantities (e.g. composition $c(\boldsymbol{x}, t)$ ) with an additional reaction-diffusion equation for the phase field $\phi(\boldsymbol{x}, t)$, often called the phase-field equation can be derived by variational derivatives

$$
\frac{\partial \phi}{\partial t}=-\mathbf{M}(\phi) \frac{\delta \mathcal{F}(\phi, c)}{\delta \phi} \quad \text { and } \quad \frac{\partial c}{\partial t}=\nabla \cdot\left\{\mathcal{D}(\phi)\left[c(1-c) \nabla\left(\frac{\delta \mathcal{F}(\phi, c)}{\delta c}\right)\right]\right\}
$$


This ansatz ensures that the total free energy decreases in time and that the concentration in the system is conserved. The derivation of the governing equations, although originally ad hoc [1], was subsequently placed in the more rigorous framework of irreversible thermodynamics $[2,3]$. The relationship of the phase-field formulation and the corresponding free-boundary problem (or sharp interface description) may be established by taking the sharp interface limit of the phase-field model, whereby the interface thickness tends to zero and is replaced by interfacial boundary conditions (see e.g. [4] and in the presence of surface energy anisotropy [5]). In [6] Karma and Rappel have developed a framework for second order sharp interface asymptotics which is more appropriate to the simulation of dendritic growth at low undercoolings by the phase-field model. In the field of solidification, phase-field models have been developed to describe both, the solidification of pure materials and binary alloys [7].

For pure materials, phase-field models have been used extensively to simulate numerically dendritic growth into an undercooled liquid, e.g. $[6,8,9,10]$. These computations exhibit a wide range of realistic phenomena associated with dendritic growth, including side arm production and coarsening. Accurate computations have been conducted at lower undercoolings closer to those encountered in experiments of dendritic growth and have also been used as a means of assessing theories of dendritic growth.

In recent years, the phase-field methodology has been extended to describe the evolution of multiple interfaces, grain boundaries and phase transitions in three phase systems. Grains in a pure material have also been modelled by multi-phase-field models using a vector valued phase field that I denote with $\phi(\boldsymbol{x}, t)=\left(\phi_{1}(\boldsymbol{x}, t), \ldots, \phi_{N}(\boldsymbol{x}, t)\right),[11,12,13,14]$. The multi-phase-field model discussed in this paper is based on an ad hoc model formulation in which a phase field is associated with each of the different phases, [12]. This model was further developed in [15] to include surface energy anisotropy, to describe and simulate grain structure formation and its sharp interface asymptotic limit was studied. In this limit the classical laws at interfaces (the Gibbs-Thomson condition) and at multi-junctions (the Young's force balance law) were recovered. Based on the same roots, the notion of a generalised $\xi$-vector formulation was used in order to incorporate anisotropy and the phase-field concept was extended to model the solidification in binary three phase alloy systems including a formulation of convection in the (monotectic) liquid phases, [16].

In this paper a thermodynamically consistent multi-phase-field model is presented which can be used to model and numerically simulate complex microstructure evolution in both, binary three phase alloy systems as well as solid/liquid phase systems with multiple crystals of different crystallographic orientation. The only difference of these two applications is that the components of the phase-field vector either represent the different phases in the alloy system or the different orientational variants of a grain configuration. 
The multi-phase-field model discussed here provides a solution of how the occurance of a third/foreign phase contribution at two phase interfaces can be avoided. We present a selection of simulations showing polycrystalline grain structures as well as phase transitions in eutectic, peritectic and monotectic alloy systems.

\section{Multi-Phase-Field Model}

The vectorial multi-phase-field model is formulated in terms of a generalized Ginzburg-Landau free energy $\mathcal{F}(\phi, c ; T)$ which is a functional of the multi-phase-field vector $\phi(x, t)=\left(\phi_{1}(\boldsymbol{x}, t), \ldots, \phi_{N}(\boldsymbol{x}, t)\right)$ and the concentration variable $c(\boldsymbol{x}, t)$

$$
\mathcal{F}(\boldsymbol{\phi}, c ; T)=\int_{V} g(\phi, \nabla \phi)+f(\phi, c ; T) d \boldsymbol{x} .
$$

For convenience, the temperature $T$ in this integral expression is treated as an external parameter, so that we discuss isothermal situations. The free energies may be given by

$$
\begin{aligned}
g(\phi, \nabla \phi) & =\sum_{i<k}^{N, N} \frac{\epsilon_{i k}^{2}}{2}\left[\Gamma_{i k}\left(\phi_{i} \nabla \phi_{k}-\phi_{k} \nabla \phi_{i}\right)\right]^{2} \\
f(\phi, c ; T) & =\sum_{i<k}^{N, N} \frac{W_{i k}}{4} \phi_{i} \phi_{k}+\sum_{i}^{N} m_{i}(c ; T) \phi_{i}+h(c)
\end{aligned}
$$

with e.g. the following choices

$$
m_{i}(c ; T)=m_{i}^{B}(T) c+m_{i}^{A}(T)(1-c) \quad \text { and } \quad h(c)=H\left(c-c^{*}\right)^{2} .
$$

Here, $m_{i}^{A}(T)$ and $m_{i}^{B}(T)$ are bulk free energies of the pure $A$ and pure $B$ states, respectively. We assume that they have the form

$$
m_{i}^{A}(T)=L^{A} \frac{T-T_{i}^{A}}{T_{i}^{A}}+m_{N}^{A}(T) \text { and } m_{i}^{B}(T)=L^{B} \frac{T-T_{i}^{B}}{T_{i}^{B}}+m_{N}^{B}(T) .
$$

The parameters $L^{A}$ and $T_{i}^{A}$ are the latent heat of fusion per unit volume and the melting temperature of the phases $i$ of pure component $A$, respectively. A similar interpretation applies to $L^{B}$ and $T_{i}^{B}$ for pure $B$. The constants $\epsilon_{i k}$ are gradient energy coefficients and $\Gamma_{i k}\left(\phi_{i} \nabla \phi_{k}-\phi_{k} \nabla \phi_{i}\right)$ are homogeneous degree one functions of their argument. As shown in [16], the quantity $\epsilon_{i k}$ is related to the interface thickness and to the surface energy of the interface between the bulk phases labelled $i$ and $k$. In particular, the surface energy anisotropy of this interface is described by the dependence of $\Gamma_{i k}$ on the orientation of its argument. If the interfaces in the system are isotropic $\Gamma_{i k}$ simplifies to $\Gamma_{i k}\left(\phi_{i} \nabla \phi_{k}-\phi_{k} \nabla \phi_{i}\right)=\left|\phi_{i} \nabla \phi_{k}-\phi_{k} \nabla \phi_{i}\right|$. 
In real physical systems, the surface energies of the individual interfaces are in general not equal. In this case, the above phase-field model and also other diffuse multi-order parameter models cited in literature suffer from the difficulty that a third or - more generally - foreign phase contribution occurs at a two phase interface, Fig. 2 a). This effect is to date interpreted to be non-physical and leads to a violation of physical laws of equilibrium thermodynamics such as the Young's force balance law at multiple junctions. Due to this disability, one does e.g. not obtain the correct angle condition at triplejunctions for a system with different surface energies. An explanation in terms of the model formulation is that the surface energies relate to the model parameters according to $\gamma_{i k} \sim \epsilon_{i k} \sqrt{W_{i k}}$, so that for different values of $\gamma_{i k}$ the model in general involves different values of the $W$ 's. As a result the shape of the hyperplane of the multi-obstacle/multi-well potential $\sum_{i<k} W_{i k} / 4 \phi_{i} \phi_{k}$ changes compared to the symmetric case of equal surface energies. The consequence is that the connecting trajectories of two minima, representing stable phase states, do no longer ly along the edges of the Gibbs simplex, but do now have a contribution towards the center involving foreign phases. An interpretation in terms of minimizing energy is that the energy of a pure two phase interface is higher than the energy of an interface involving small amounts of other foreign phases. A possible solution of this difficulty is the introduction of higher order potentials of the form $\sim \phi_{i} \phi_{j} \phi_{k}$ (see [15]) which increase the energy of the center of the Gibbs simplex and which force the third/foreign phase to vanish at a two phase interface, Fig. 2 b). These higher order potentials have no physical meaning and enhance the complexity of the model formulation.
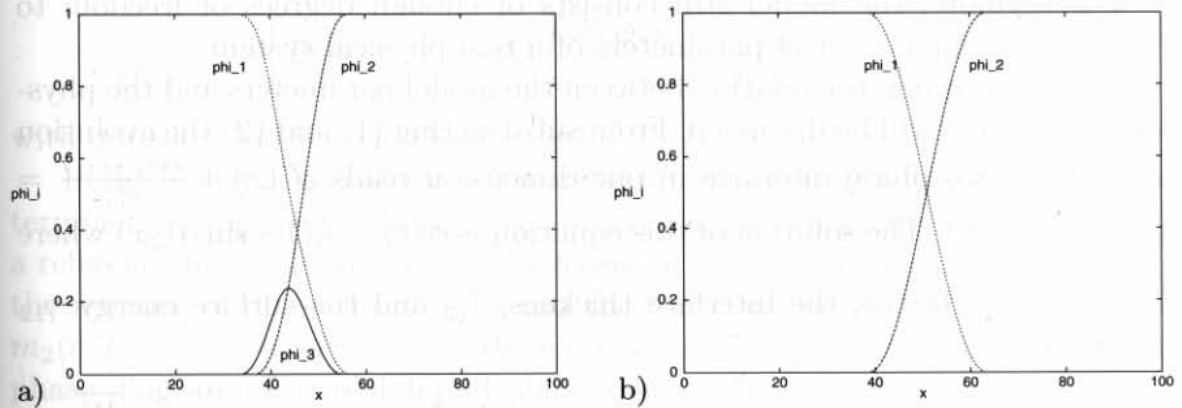

Fig. 2. a) Contour lines of three phase-field variables at a two phase boundary in a three phase system for different surface energies and by using a) the multi-obstacle potential $\frac{W_{i k}}{4} \phi_{i} \phi_{k}$ and b) the multi-obstacle potential with additional higher order correction terms $\sim \phi_{i} \phi_{j} \phi_{k}$. The plot in a) illustrates the presence of a third/foreign phase whereas in b) only the two appropriate phases participate in forming the boundary 
In the following an alternative solution avoiding the third/foreign phase contribution at two phase interfaces is proposed. We use the notation

$$
\nabla_{i k}:=\phi_{i} \nabla \phi_{k}-\phi_{k} \nabla \phi_{i} \quad \text { and } \quad \triangle_{i k}:=\phi_{i} \nabla^{2} \phi_{k}-\phi_{k} \nabla^{2} \phi_{i}
$$

Next we consider a two phase interface of phases labelled 1 and 2 with $\phi_{3}=0$ in a three phase system. At this interface the evolution equations are

$$
\begin{aligned}
\epsilon_{12}^{2}\left(\phi_{2} \triangle_{12}+2 \nabla_{12} \cdot \nabla \phi_{2}\right)+ & \frac{1}{4}\left(W_{12} \phi_{2}\right)+m_{1}(c, T)-\lambda=0 \\
\epsilon_{12}^{2}\left(-\phi_{1} \triangle_{12}+2 \nabla_{12} \cdot \nabla \phi_{1}\right)+ & \frac{1}{4}\left(W_{12} \phi_{1}\right)+m_{2}(c, T)-\lambda=0 \\
\frac{1}{4}\left(W_{13} \phi_{1}+W_{23} \phi_{2}\right)-\lambda & =0
\end{aligned}
$$

where $\lambda$ is a Lagrangian multiplier. From the third equation, the equality $W_{13}=W_{23}$ follows as a necessary condition for $\lambda$ to be constant. For an $N$ phase system, we obtain $W_{i k}=: W$ for all $i, k=1, \ldots, N$. Subsequently, the solution to overcome the difficulties of the third/foreign phase contribution at two phase interfaces is to keep the multi-obstacle potential $\sum_{i<k} \frac{W_{i k}}{4} \phi_{i} \phi_{k}$ fixed and independent of the surface energies. As a result, the shape of the hyperplane of $\sum_{i<k} \frac{W_{i k}}{4} \phi_{i} \phi_{k}$ is not influenced by any system parameters and does not change. Therefore, the above expression for the free energy contribution $f(\phi, c, T)$ of the multi-phase-field model is replaced by

$$
f(\phi, c ; T)=\sum_{i<k}^{N, N} \frac{W}{4} \phi_{i} \phi_{k}+\sum_{i}^{N} m_{i}(c ; T) \phi_{i}+h(c) .
$$

Furthermore, the model still consists of enough degrees of freedom to realize the complete set of parameters of a real physical system.

In the following, the relation between the model parameters and the physical parameters will be discussed. From substracting (1) and (2) the evolution of a single two phase interface in one dimension reads $\phi^{\prime \prime}(x)+\frac{\left(m_{2}-m_{1}\right)}{\epsilon_{12}^{2}}=$ $\frac{W}{4 \epsilon_{12}^{2}}(1-2 \phi(x))$. The solution of this equation is $\phi(x)=\frac{1}{2}\left(1+\sin \alpha_{12} x\right)$ where $\alpha_{12}=\frac{\sqrt{W / 2}}{\epsilon_{12}}$. Hence, the interface thickness $l_{12}$ and the surface energy $\gamma_{12}$ relate to $\epsilon_{12}$

$$
l_{12}=\frac{\pi}{2 \alpha_{12}}=\frac{\pi \epsilon_{12}}{\sqrt{2 W}} \quad \text { and } \quad \gamma_{12}=\frac{1}{2} \int_{l_{12}} \phi(1-\phi) d x=\frac{\pi}{8} \sqrt{\frac{W}{2}} \epsilon_{12}
$$

The interface mobility $\mu_{12}$ is

$$
\mu_{12}=\frac{-M_{12} \int_{0}^{1} m(c ; T) d \phi}{\int_{l_{12}} \phi^{\prime}(x) d x}=\frac{-8 C}{\pi^{2}} M_{12} l_{12}
$$

with $m(c ; T)=m_{1}(c ; T)=m_{1}^{B}(T) c+m_{1}^{A}(T)(1-c)$ and $m_{2}(c ; T)=0$ for the two phase interface in equilibrium and where $M_{12}$ is the mobility matrix. 
The constant $C$ is derived from the equilibrium concentration equation at the interface

$$
\frac{\partial f(\phi, c ; T)}{\partial c}=\left(m_{1}^{B}(T)-m_{1}^{A}(T)\right) \phi+2 H\left(c-c^{*}\right)-\lambda_{c}=0
$$

where $\lambda_{c}$ is a Lagrange multiplier. It follows that

$$
\begin{aligned}
c & =\frac{1}{2 H}\left[\lambda_{c}-\left(m_{1}^{B}-m_{1}^{A}\right) \phi\right]+c^{*} \text { and } \\
m(c ; T) & =\left(m_{1}^{B}-m_{1}^{A}\right) c+m_{1}^{A} \\
& =-\frac{1}{2 H}\left(m_{1}^{B}-m_{1}^{A}\right)^{2} \phi+\left(\frac{1}{2 H} \lambda_{c}+c^{*}\right)\left(m_{1}^{B}-m_{1}^{A}\right)+m_{1}^{A} \\
& =: C_{1} \phi+C_{2} .
\end{aligned}
$$

The definition for $C$ is $C:=\frac{1}{2} C_{1}+C_{2}$.

Starting from a real system, the recepy for choosing the model parameters is; first, take suitable values for $\epsilon_{i k}$ to represent the surface energies $\gamma_{i k}$. This choice of $\epsilon_{i k}$ then adjusts certain interface thicknesses $l_{i k}$. To realize physical mobilities $\mu_{i k}$, it is now necessary to choose the values of the mobility matrix $M_{i k}$ in an appropriate way.

The presented phase-field model is capable to describe both, binary three phase alloy systems such as eutectics, peritectics and monotectics as well as solid/liquid phase systems with an arbitrary number of crystals of different crystallographic orientation. We postulate the set of evolution equations for the phase fields $\phi_{i}(\boldsymbol{x}, t)$ and for the concentration $c(\boldsymbol{x}, t)$ from the gradient flow of the energy functional

$$
\begin{aligned}
\frac{\partial \phi_{i}}{\partial t} & =-\mathbf{M}(\phi) \frac{\delta \mathcal{F}(\phi, c ; T)}{\delta \phi_{i}}, \quad i=1, \ldots, N \\
\frac{\partial c}{\partial t} & =\nabla \cdot\left\{\mathcal{D}(\phi)\left[c(1-c) \nabla\left(\frac{\mathcal{F}(\phi, c ; T)}{\delta c}\right)\right]\right\}
\end{aligned}
$$

with diffusion coeffictions $\mathcal{D}(\phi)=\frac{v_{m}}{R T} \sum_{i=1}^{N} D_{i} \phi_{i}$.

In the binary alloy case $N=3$ and the functions $m_{1}(c ; T), m_{2}(c ; T)$ are determined by the specific form of the phase diagram choosing $m_{3}(c, T)=0$ as a reference frame. Examples can be found in [16]. In the crystal growth case, the number of phase fields is $N$ and the functions $m_{i}(c ; T)$ are $m_{1}(c ; T)=$ $m_{2}(c ; T)=\ldots=m_{N-1}(c)$ with $m_{N}(c ; T)=0$ representing a lense shape phase diagram of a solid/liquid phase system. In addition the diffusion coefficients of all solid crystals are equal $D_{1}=\ldots=D_{N-1}$. They are approximately three orders of magnitude less than in the liquid phase labelled $N$; $D_{i}<<D_{N}, i=1, \ldots, N-1$.

\section{Numerical Simulations}

The objective of this section is to demonstrate the general utility of the multi-phase-field model as a computational vehicle to simulate a wide variety of grain growth structures and realistic solidification morphologies in 
peritectic, eutectic and monotectic alloy systems. Pursuing this aim, a selection of numerical solutions of the set of partial differential equations for the phase-field vector $\boldsymbol{\phi}(\boldsymbol{x}, t)$ and for the concentration $c(\boldsymbol{x}, t)$ is presented. A finite difference discretisation on a uniform rectangular mesh allied to an explicit time marching scheme is used for the three phase-fields and for the solute concentration. Effects of fluid flow have not been taken into account. Further details concerning the numerical setup and the system parameters are given in [15].

\subsection{Grain Structures}

First, we apply the multi-phase-field model to grain growth phenomena. In this context, the components of $\phi(x, t)$ represent grains of the same phase, but of different crystallographic orientations. The simulation depicted in Fig. 3 is performed for a microstructure with four orientational variants and with a sixfold convex crystalline surface energy anisotropy. The incorporation of surface energy anisotropy and explicit expressions for crystalline anisotropy with a typical cusp-like structure are explained in [15]. The evolution of the grain boundaries in Fig. 3 shows the coarsening of the grain structure and the formation of facets in the six preferred directions of the Wulff shape. The anisotropy of the surface energies induces shear forces which change the equilibrium angle condition of $120^{\circ}$.

In isotropic systems, wetting occurs if the surface energies violate the stability condition $\gamma_{i j} \leq \gamma_{i k}+\gamma_{k j}$. Fig. 4 illustrates computations of wetting for a system of four different phase fields. The time sequence in Fig. 4 a) and $b$ ) shows the break down of 123 and 234 grain boundary triple junctions accompanied by the building of channels of the grains 1 and 4 between grain
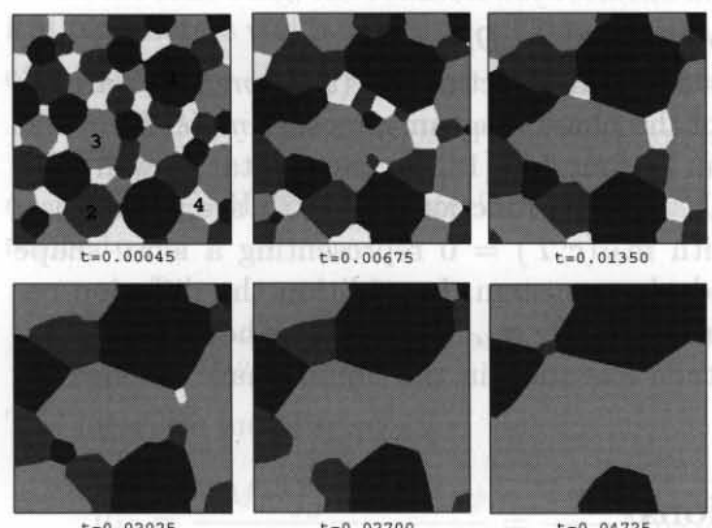

Fig. 3. Growth of grains with a six-fold anisotropy and with relative angles: $\varphi_{12}=$ $30^{\circ}, \varphi_{13}=45^{\circ}, \varphi_{14}=90^{\circ}, \varphi_{23}=15^{\circ}, \varphi_{24}=60^{\circ}, \varphi_{34}=45^{\circ}$ 
(a)
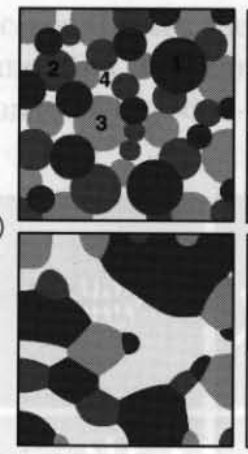
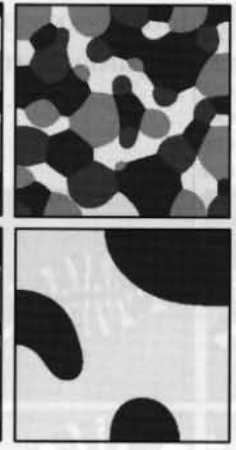

(b)

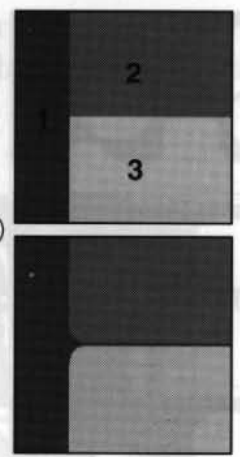

Fig. 4. Wetting in an isotropic system with surface energies $\gamma_{12}=\gamma_{13}=\gamma_{14}=$ $\gamma_{24}=\gamma_{34}=0.7$ and $\gamma_{23}=1.8$

2 and grain 3 . As a result, the grains remaining last in the coarsening process of Fig. 4 a) are the grains 1 and 4 .

\subsection{Multiphase Alloy Systems}

Next, the phase-field method is used to numerically simulate phase transitions in binary metallic alloys. The following computations of multiphase solidification are carried out under the condition of an isothermally undercooled melt and for isotropic surface energies. Further details concerning the numerical setup and the system parameters are provided in [16].

First, we present a numerical simulation corresponding to peritectic solidification. In a peritectic system, cooled beneath its peritectic temperature, a new solid phase $\beta$ nucleates, i.e., $L+\alpha \rightarrow \beta$. It grows from the undercooled liquid $L$ and the parent solid phase $\alpha$. By heterogeneous nucleation, the $\beta$ phase often occurs at the $\alpha-L$ interface. The $\beta$ phase then grows around the $\alpha$ phase until, either the $\alpha$ phase is completely melted or it is entirely engulfed within the new $\beta$ phase. For the computation in Fig. 5, we initially placed circular $\alpha$ solid phase particles of different radii in the liquid region and within the vicinity of a $\beta$ phase planar front. The solidification velocity of the growing $\beta$ phase is locally higher in regions close to the melting $\alpha$ particles due to locally different concentration gradients in the liquid phase. As a result, the initially planar $\beta$ front deformes due to preferential growth towards the solid particles. Triplejunctions of all three phases lead the growth direction, because the $\alpha$ phase provides the supply of solute needed for the growth of the $\beta$ phase. Depending on the size of the $\alpha$ particles and on the solidification velocity of the $\beta$ phase, the $\alpha$ particles either dissolve in the liquid or become engulfed in the $\beta$ phase. Since we assume stoichiometric solid phases, the solid diffusivity is zero and the $\alpha$ particles remain stable in size after engulfment in the $\beta$ matrix. 

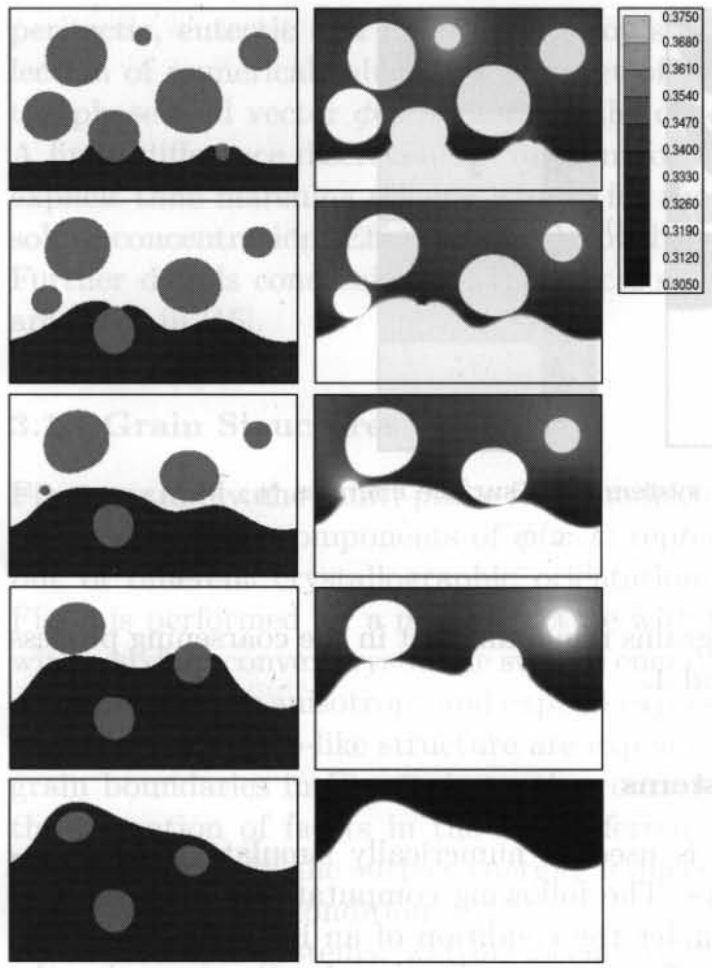

Fig. 5. Engulfment of properitectic $\alpha$ solid particles into a growing $\beta$ solid front following a peritectic phase transition $L+\alpha \rightarrow \beta$. The plots show the temporal evolution of the phase configuration and the concentration profile in the liquid phase in the left and right column, respectively. In this calculation, diffusion in the solid phases is suppressed, $D_{1}=D_{2}=0$

Next, the result of a numerical simulation corresponding to eutectic solidification is reported. In particular, the specific data of the $A l-S i$ phase diagram are used for the computation in Fig. 6. Once the metallic melt in a eutectic system is cooled down below a critical temperature, the liquid phase $L$ transforms into two new solid phases $\alpha$ and $\beta$ via a eutectic reaction: $L \rightarrow \alpha+\beta$. The black and white regions in the images of Fig. 6 represent the $A l$ - and $S i$ - rich eutectic solid phases $\alpha$ and $\beta$ of the binary $A l-S i$ alloy system, respectively. The snapshots show the concentration field of $S i$ in the liquid during the growth of a eutectic grain at different time steps. We observe zones of depleted solute ahead of the $S i$-rich solid phase and concentration enriched regions ahead of the $A l$-rich solid phase. Due to an increase of the lamellar spacing during growth, deep concave hollows are formed at the solid-liquid interfaces and the phase boundaries of the eutectic microstructure evolve in a disordered manner. The subsequent nucleation of solid particles of the opposite phase within the concave portion of the inter- 
faces stabilizes the growth behaviour and re-establishes lamellar growth. The simulated structure is compared with an experimental photograph in the last image of Fig. 6.
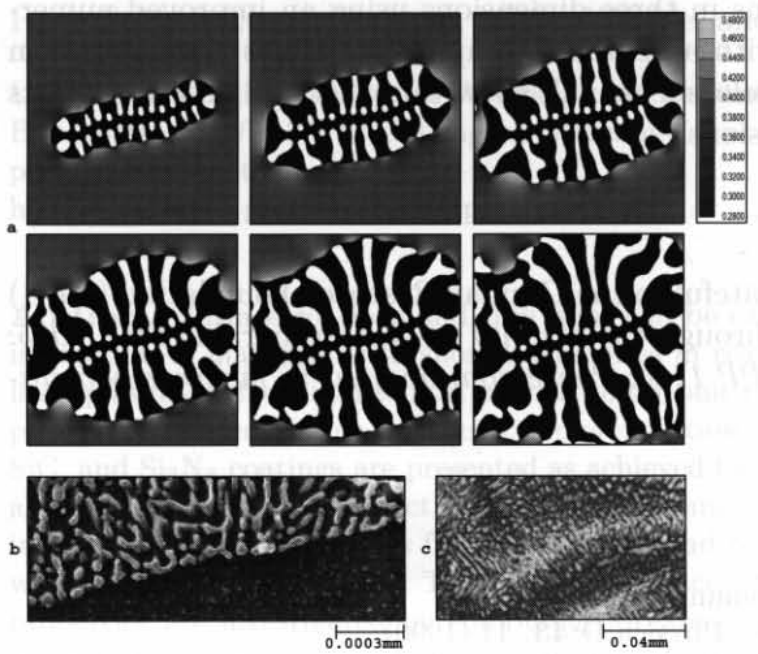

Fig. 6. Phase-field simulation of a eutectic $A l-S i$ grain in comparison with experimental photographs. The snapshots of the calculation display the concentration of silicon and the evolution of the phase boundaries propagating in time

\section{Conclusion}

The multi-phase-field model discussed here has been derived in a thermodynamically consistent way and shows a way of how a third/foreign phase contribution along a two phase interface can be avoided. Numerical simulations of grain boundary motion and binary three phase solidification are presented which illustrate the general capability of the model to describe realistic microstructure evolutions.

As a next step of progress, we are recently working on an extension of the above multi-phase-field model to multicomponent (ternary) multiphase alloys. To treat the phase transformations in an $M$-component alloy system with $M+1$ different phases, a vector $\mathbf{c}=\left(c_{i}, \ldots, c_{M}\right)$ of $M$ concentration fields $c_{i}, i=1, \ldots, M$ is introduced, so that the energy functional $\mathcal{F}(\phi, \mathbf{c}, T)$ now also depends on $\mathbf{c}$. The governing equations are complemented by a set of $M$ differential equations for the diffusion fields. This model extension will be used to describe the growth of eutectic colonies in ternary eutectic systems. Another important objective is the investigation of multiscale solidification phenomena such as primary dendrites with interdendritic eutectic regions. 
Within this context, the aim is to derive new growth laws on larger length scales by homogenization and averaging the temporal evolution of the structure on finer scales. Furthermore, it is intended to incorporate convection and elasticity into the phase-field model. Based on the promising numerical results in 2 dimensions, simulations in three dimensions using an improved numerical algorithm combined with acceptable computation times are planned in our future work. These developments will allow to reflect and describe effects due to three spatial dimensions.

\section{Acknowledgements}

The author B. Nestler is grateful to the German Research Foundation (DFG) for supporting this work through the priority research programs SPP 1095: Mehrskalenprobleme and SPP 1120: Phasenumwandlung in mehrkomponentigen Schmelzen.

\section{References}

1. J. S. Langer, private communication.

2. O. Penrose and P. C. Fife, Physica D 43, 44 (1990).

3. S-L Wang, R. F. Sekerka, A. A. Wheeler, B. T. Murray, S. R. Coriell, R. J. Braun, G. B. McFadden, Physica D 69, 189 (1993).

4. G. Caginalp, Phys. Rev. A 39, 5887 (1989).

5. A. A. Wheeler and G. B. McFadden, Eur. J. Appl. Math. 7, 369 (1996).

6. A. Karma and W.-J. Rappel, Phys. Rev. E 53, R3017 (1996).

7. A. A. Wheeler, W. J. Boettinger, G. B. McFadden, Phys. Rev. A 45, 7424 (1992); A. A. Wheeler, W. J. Boettinger, G. B. McFadden, Phys. Rev. E 47, 1893 (1993); J. A. Warren and W. J. Boettinger, Acta. metall. mater. 43, 689 (1994).

8. R. Kobayashi, Bull. Jpn. Soc. Ind. Appl. Math. 1, 22 (1991); R. Kobayashi, Physica D 63, 410 (1993); R. Kobayashi, Experimental Math 3, 60 (1994).

9. S-L. Wang and R. F. Sekerka, J. Comp. Phys. 127, 110 (1996).

10. N. Provatas, N. Goldenfeld, and J. Dantzig, Phys. Rev. Lett. 80, 3308 (1998)

11. Long-Qing Chen and Wei Young, Phys. Rev. B 50, 15752 (1994); Long-Qing Chen, Scr. Metall. Mater. 32, 115 (1995).

12. I. Steinbach, F. Pezzolla, B. Nestler, J. Rezende, M. Seesselberg and G. J. Schmitz, Physica D 94, 135 (1996).

13. B. Nestler and A. A. Wheeler, Phys. Rev. E 57, 2602 (1998).

14. R. Kobayashi, J. A. Warren, and W.C. Carter, Physica D 119, 415 (1998).

15. H. Garcke, B. Nestler and B. Stoth, Physica D 115, 87 (1998); H. Garcke, B. Nestler and B. Stoth, SIAM Journal on Applied Mathematics 60, 295 (1999); H. Garcke, B. Nestler and B. Stoth, J. Interfaces and Free Boundary Problems 1, 175 (1999); H. Garcke and B. Nestler, Mathematical Models and Methods in Applied Sciences 10, 895 (2000).

16. B. Nestler and A. A. Wheeler, Phys. Rev. E 57, 2602 (1998); B. Nestler and A.

A. Wheeler, Physica D 138, 114 (2000); B. Nestler, A. A. Wheeler, L. Ratke, C. Stöcker, Physica D 141, 133 (2000). 